UNIT NO. 2

\title{
USING THE SBAR4 MODEL FOR MANAGEMENT OF A PATIENT WITH COMPLEX COMORBIDITIES IN THE COMMUNITY HOSPITAL — A CASE STUDY
}

Dr Xu Bangyu, Dr Low Sher Guan Luke

\begin{abstract}
Patients who require a stay in a community hospital usually tend to be more complex, presenting not only with biomedical issues with complications, but also with a myriad of psychological and social issues as well. If they were to be discharged from an acute hospital directly to primary care and community, the patients and caregivers may feel helpless, overwhelmed and unsure of how to navigate the healthcare system to get their complex issues sorted out. Family physicians in the community hospitals need to hone their skills in such an area of care. The SBAR4 model can be effectively used to categorise the patients' multiple bio-psycho-social issues, coordinate the multi-disciplinary team to bring hospital and community resources to help such patients, provide holistic care for such patients, and transit them safely into the care of our family physicians in primary care and community.
\end{abstract}

Keywords: Complex Care; Community Hospital; SBAR4; Holistic Care; Multi-Disciplinary;

SFP2017; 43(3) : I I-I5

\section{CASE VIGNETTE AND SITUATION}

Madam P is a 57-year-old Chinese lady who was transferred from Hospital A to Sengkang Health (SKH) after an episode of acute pontine infarct, for stroke rehabilitation. During her stay with us, we picked up additional issues of frailty, osteoporosis, caregiver stress as well as post-stroke depression which resulted in poor progress in her rehabilitation. Through this case vignette, we hope to demonstrate the SBAR4 model in holistically managing her complex care and planning for her transition back to the community.

\section{BACKGROUND}

Madam P had multiple cardiovascular risk factors of Diabetes Mellitus (DM) for 15 years, complicated by Peripheral Vascular Disease (PVD) with Right Below-Knee Amputation (BKA) done for right foot gangrene in March 2015. In addition, she had hypertension complicated by previous left corona radiata and thalamic infarcts in June 2013, with good functional recovery and hyperlipidaemia.

She is ADL-independent, ambulates with a right prosthesis, and is on follow-up with XX Polyclinic for her chronic diseases. She is a full-time housewife and she neither smokes nor drinks

\section{XU BANGYU}

Senior Staff Registrar, Department of Family Medicine,

Sengkang Health, Singhealth

\section{LOW SHER GUAN LUKE}

Consultant, Department of Family Medicine,

Sengkang Health alcohol. She stays with her husband and son.

Her list of medications is as follows:

1. Aspirin 100mg OM

2. Atorvastatin $40 \mathrm{mg} \mathrm{ON}$

3. Glipizide $12.5 \mathrm{mg} \mathrm{OM} 7.5 \mathrm{mg} \mathrm{ON}$

4. Metformin $1 \mathrm{~g} \mathrm{BD}$

5. Omeprazole $20 \mathrm{mg}$ OM

6. Amlodipine 5mg OM

7. Losartan $100 \mathrm{mg} \mathrm{OM}$

8. Tears Naturale eye drops 2 drops QDS to right eye

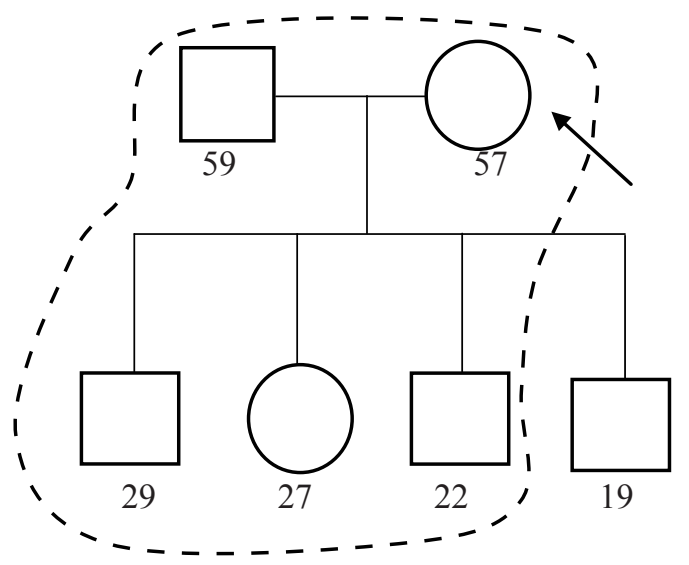

She was admitted to Hospital A Neurology for 2 weeks for acute pontine infarct. She presented with persistent giddiness and a new left-sided internuclear ophthalmoplegia was detected during physical examination. During the admission in Hospital A, she was started on dual antiplatelet therapy for 3 weeks followed by lifelong clopidogrel. She was transferred to Sengkang Health (SKH) for stroke rehabilitation.

\section{Admission to SKH for Rehabilitation}

Madam P looked depressed during this admission. She was teary and in low mood when giving her account of her clinical history. Unfortunately, the support she received from her family members was poor and she felt depressed. Her appetite was poor and she had anhedonia. She complained of insomnia with frequent awakening. She did not have any active suicidal thoughts or hallucinations. She also had persistent non-vertiginous giddiness since this new episode of stroke. This was not associated with hearing loss or tinnitus. It was also not related to her posture. She did not have any cardiac-related complaints of chest pain, shortness of breath, or palpitation. Her husband appeared to be stressed out, having asked multiple times for her to be sent to a nursing home as he had difficulty coping. As such, she lost all motivation and felt that rehabilitation would be pointless. 


\section{CLINICAL ASSESSMENT}

On examination, she was afebrile and her supine BP was $145 / 95 \mathrm{mmHg}$, confirming suboptimal control of existing hypertension. Her standing BP 3 minutes later was $138 / 90 \mathrm{mmHg}$ with no significant postural drop. Her BMI was low at $17.1 \mathrm{~kg} / \mathrm{m}^{2}$ as she had a right BKA. Her random capillary blood glucose (CBG) was $16.8 \mathrm{mmol} / \mathrm{L}$. Her resting pulse rate was regular in rhythm at $80 / \mathrm{min}$, not in atrial fibrillation. Right upper motor neuron (UMN) facial nerve palsy was seen with power of $4 / 5$ in both right upper and lower limbs with brisk tendon reflexes. She had a right pronator drift. On right lateral gaze, there was failure of adduction of her left eye with nystagmus noted in her right eye. These findings were absent on left lateral gaze, confirming the presence of left internuclear ophthalmoplegia. Visual field assessment did not detect any abnormalities and both pupils were reactive and equal to light with preserved direct and consensual pupillary reflexes. No other cranial nerve palsies were detected. She did not have dysdiadochokinesia, dysmetria, or dysarthria. Joint proprioception was impaired in her left foot. Her bedside swallowing test was normal. Examination of her cardiorespiratory systems was unremarkable. No carotid bruits were detected as well. Her right knee stump was healthy, with no abrasions or pressure sores seen. Her hand grip strength was $12 \mathrm{~kg}$. Her bedside Abbreviated Mental Test (AMT) was 10/10. Her Mini-Mental State Examination (MMSE) was 29/30 and 7-item Geriatic Depression Scale (GDS) was more than 5, suggestive of depression.

Table 1: Laboratory results traced via NEHR records.

\begin{tabular}{|c|c|c|}
\hline & Patient & Reference range \\
\hline TC & 5.85 & $<5.2 \mathrm{mmol} / \mathrm{L}$ \\
\hline TG & 2.17 & $<1.70 \mathrm{mmol} / \mathrm{L}$ \\
\hline HDL & 1.25 & $>0.9 \mathrm{mmol} / \mathrm{L}$ \\
\hline LDL & 2.61 & $<2.6 \mathrm{mmol} / \mathrm{L}$ \\
\hline Sodium & 138 & $135-145 \mathrm{mmol} / \mathrm{L}$ \\
\hline Potassium & 3.9 & $3.5-5.0 \mathrm{mmol} / \mathrm{L}$ \\
\hline Urea & 2.8 & $2.7-6.9 \mathrm{mmol} / \mathrm{L}$ \\
\hline Creatinine & 97 & $37-75 \mu \mathrm{mol} / \mathrm{L}$ \\
\hline HbA1c & $10.3 \%$ & $<7.0 \%$ \\
\hline
\end{tabular}

In view of the low mood, we needed to exclude organic causes such as hypothyroidism. As a result of her new stroke with right-sided weakness, as well as her existing right BKA, her fall risk was higher, so we sought to exclude any existing osteoporosis and vitamin D deficiency. The fact that she was largely homebound and was rarely out in the sun put her at higher risk for vitamin $\mathrm{D}$ deficiency as well. The results are shown below:

Table 2: Results of tests to exclude organic causes.

\begin{tabular}{|c|c|l|}
\hline & \multicolumn{1}{|c|}{ Patient } & \multicolumn{1}{c|}{ Reference range } \\
\hline 25(OH)D & 10.9 & \multicolumn{1}{c|}{$30-100 \mathrm{NG} / \mathrm{ML}$} \\
\hline Free T4 & 13.4 & $8.8-14.4 \mathrm{PMOL} / \mathrm{L}$ \\
\hline TSH & 0.269 & $0.65-3.70 \mathrm{MU} / \mathrm{L}$ \\
\hline BMD & $\begin{array}{c}\text { T score }<-4.1 \text { for left hip } \\
\text { T score }<-2.3 \text { for lumbar spine }\end{array}$ & $\begin{array}{l}\text { 1. T-score greater than }-1 \text { considered normal. } \\
\text { 2. T-score between }-1 \text { and }-2.5 \text { indicates } \\
\text { osteopenia. } \\
\text { 3. T-score below }-2.5 \text { indicates osteoporosis } \\
\text { even without concomitant fracture. } \\
\text { 4. Patients with osteoporotic fractures are } \\
\text { considered to have established or severe } \\
\text { osteoporosis. }\end{array}$ \\
\hline
\end{tabular}

From the above thorough assessment, we teased out and prioritised Madam P's problems into 3 major domains to make it easier to deal with each one of them.

\section{Biomedical Issues}

1. Poor rehabilitation progress post-stroke (at level of left medial longitudinal fasciculus)

Likely contributed by depression and existing cardiovascular risk factors, especially poorly controlled DM.

2. High fall risk

- $\quad$ Made worse by recent stroke causing mild right hemiparesis.

Contributed by right BKA affecting balance and impaired joint proprioception from chronic DM.

3. Frailty, osteoporosis with vitamin D deficiency Contributed by multiple chronic diseases and sarcopenia.

Potentially increases risks for fragility fractures coupled with the above high fall risk.

4. Existing high cardiovascular risk factors Poorly controlled DM with complications.

Suboptimal control of hypertension. Suboptimal control of hyperlipidemia.

\section{Psychological Issues}

\section{Post-stroke depression}

- $\quad$ Met the criteria for depression (low mood, poor appetite, sleep disturbance, loss of hope) but not actively suicidal.

- Contributed by recent new stroke, decline in function and poor family support. Resulting in poor motivation for rehabilitation, hindering functional improvement.

Exacerbated by the above negative reinforcement vicious cycle and causing feelings of hopelessness.

\section{Social Issues}

6. Adverse social circumstances

Contributed by caregiver stress and inability to cope, poor family support, and financial difficulties, with mention of sending Madam P to a nursing home for which she was not agreeable, thus worsening family dynamics.

\section{RECOMMENDATIONS, RESOURCES, RESPONSIBILITY, AND RELATIONSHIP}

From the above problem list, we individualised a comprehensive plan for Madam P which included our recommendations for the various issues, bringing together hospital and community 
resources to help both the patient and her family, educating and empowering the patient with the appropriate responsibility for her care, as well as exploring relationships between Madam $\mathrm{P}$ and various stakeholders such as family and physicians that can potentially impact on her care.

\section{Poorly Controlled DM}

Mdm P had been non-compliant with her medications for the previous 6 months. She lost the motivation after her right BKA in March 2015. She did not follow the dietitian's advice as well. She had defaulted on her follow-up with XX polyclinic on a few occasions when her family members were not able to accompany her. Her Ideas, Concerns, and Expectations (ICE) were explored. Her poor compliance to treatment and follow-ups had resulted in disease complications. Working together with our dietitian and Diabetic Nurse Educator, we managed to change her knowledge, attitudes, and perceptions of the long-term care of DM after multiple counselling sessions. Mdm P became compliant to her medication and DM diet, and her in-patient CBG trend demonstrated improvement in glycaemic control. As she had defaulted on her yearly diabetic retinopathy and foot screening, these were performed during her current inpatient stay, and the appropriate eye, left foot and right stump care advice was given.

\section{Depression}

Mirtazapine $15 \mathrm{mg}$ ON was started for treatment of her depression. Working together with the ward nurses, psychologist, and physio-/occupational therapists, we provided motivation and encouragement for Mdm P constantly. The ward nurses arranged for her to be brought out of the ward for sunlight exposure to improve her mood. Mdm P was advised to practice good sleep hygiene to improve her insomnia. Her dose of mirtazapine was slowly titrated up to $30 \mathrm{mg} \mathrm{ON}$ with gradual improvement in her mood.Her serum sodium level was monitored and no hyponatremia was detected. Her Electrocardiogram (ECG) also did not show prolonged QTc. With these interventions, her repeated GDS had improved and her score was less than 5 .

\section{Stroke, Frailty, Osteoporosis and Vitamin D Deficiency}

Initially, Mdm P was not compliant with rehabilitation sessions due to her depressed mood. As her mood improved with treatment, her compliance to rehabilitation sessions also improved. With multiple sessions of isometric, concentric, and eccentric exercises, her hand-grip strength slowly improved from 12 to $30 \mathrm{~kg}$. Her FIM score also improved with the rehabilitation sessions. Her giddiness gradually resolved as she continued to participate in therapy sessions. Together with the therapists, we constantly encouraged her and educated her about the importance of compliance to rehabilitation sessions and her chronic disease medications. Mdm $\mathrm{P}$ was educated about the detrimental effects of frailty and various community resources to help her tackle frailty upon discharge were made known to her. Mdm P's 25(OH)D level was low at 8.9 and Ergocalciferol
50000IU weekly for 8 weeks was initiated, followed by colecalciferol 1000 IU OM thereafter.

\section{High Fall Risk}

On assessing Mdm P's artificial limb, it was found to be ill-fitting to her right BKA stump. Arrangement was made with the Artificial Limb Centre to have the earliest possible appointment for Mdm P to change her artificial limb. In the meantime, the decision was made for $\mathrm{Mdm} \mathrm{P}$ to mobilize in a wheelchair for the time being while awaiting for her appointment. The occupational therapists did her home assessment and we activated the Enhancement for Active SEniors (EASE) programme to help with home modification and improvement to ensure a safer home environment.

\section{Adverse Social Circumstances, Caregiver Stress, Financial Difficulties}

Mdm P's husband had expressed fatigue and stress when taking care of her. He was no longer working and had no income. Their children were working most of the time and were only home during the late evening. They had a strained relationship with their youngest son, who had moved out of the house. Her husband had difficulty coping and hence requested for Mdm P to be sent to a nursing home. As Mdm P did not agree to the option of nursing home placement, this had led to further strain in their relationship. The Medical Social Worker (MSW) explored the various options that were available to them. A few family conferences were held together with the MSW and eventually, Mdm P and her family decided to engage a maid. During this period of time, Mdm P's husband was also managed apprioriately to ensure that he did not have symptoms of depression. The MSW also helped with their financial difficulties through means testing and activating the appropriate healthcare subsidies.

\section{Planning for Discharge}

After 4 weeks of stay, Mdm P had made improvements to her health. Her Functional Independence Measure (FIM) score had improved and achieved FIM efficiency plateau. She was compliant with her diet and chronic disease medications, and her inpatient CBG had been optimal. The MSW also worked with her family members and the application for a maid was successful. The multidisciplinary team's recommendation was for $\mathrm{Mdm} \mathrm{P}$ to continue outpatient rehabilitation sessions in order to tackle frailty and also to minimize deconditioning. As Mdm P's care was complex, an early review at our SKH Family Medicine Clinic to consolidate all her care plans and to follow-up her various conditions was arranged before discharging her back to XX polyclinic for chronic care.

Do take note of how the above management has seamlessly woven in the R4 (recommendations, resources, responsibility, relationship) approach which restores context to the inter-related problems and reconstitutes the fragmented information. The result is a comprehensive and continuing care plan for Mdm P. 
The breakdown of the $\mathrm{R} 4$ components is as follows:

\section{Recommendations}

For every bio-psycho-social problem listed above, the family physician and his team made appropriate recommendations and took the best course of action for Mdm P, such as adherence to her diabetic diet, and need for compliance to medications and follow-ups. To the healthcare team, these recommendations may be common sense, but to the layperson, these recommendations may be overwhelming. As such, they should always be explained clearly to the patients and caregivers so that they receive clear instructions on how to manage their health rather than be overwhelmed.

\section{Resources}

For Mdm P, a myriad of hospital and community health and social resources were roped in to help her. These included the EASE programme, financial support, adjustment of prosthesis from Artificial Limb Centre, outpatient rehabilitation therapy, maid agency, diabetic nurse education, mood counseling, and even the XX polyclinic for continued long-term care of Mdm P. Family physicians need an intricate knowledge of such resources in the hospital and community in order to effectively bring them onboard and help patients navigate the complex healthcare and social landscape during care transitions and long-term care.

\section{Responsibility}

Mdm P was educated on the clinical impact of diabetes and empowered to take personal responsibility of her own health, rather than give up in despair and wallow in hopelessness. The healthcare team members also needed to be sure of their individual roles and discharge their responsibilities, bringing their unique knowledge and skillsets to help their patients at different points and aspects of their healthcare journey. Such a diverse team needs to be led by family physicians who take charge, bear the responsibility for the care of the patient, have complete oversight of the patient's problems, and coordinate the different team members with a common goal of holistic care for the patient.

\section{Relationship}

Through in-depth history-taking, counselling, and ongoing therapeutic relationships, family physicians and their team are in the best position to explore relationship issues as these may either be the cause of, or the result of, existing biomedical issues. In Mdm P's case, her right BKA increased her dependency on her husband, who in turn was stressed and this made Mdm P feel that the support from him was not enough. This led to relationship strain and a vicious cycle of family tension and conflicts. Mdm P's husband obviously did not cope well alone and needed help. With a new maid coming onboard and sharing the caregiver burden, he was able to get occasional respite, take better care of himself and, in turn, take better care of Mdm P. This would translate to Mdm P's physical needs being better met and her mood improved. Resolving such relationship issues had helped Mdm P with her biomedical and psychological issues.

\section{CONCLUSION}

Our patients as well as the healthcare system are becoming increasingly complex and family medicine training has been evolving to keep up with the pace. Family physicians, especially those at the consultant level in the community hospitals, need to have oversight of their patient's problem list and management plans. The SBAR4 model is proposed as a useful tool for clinical use in order to have a comprehensive and integrative approach to managing such patients in the community hospital setting. We hope that, by demonstrating the use of the SBAR4 model here, it will no longer be seen as just another concept, but a useful tool that can be easily operationalised by trained family physicians and their teams.

\section{REFERENCES}

I. Png ME, Yoong J, Phan TP, Wee HL. Current and future economic burden of diabetes among working-age adults in Asia: conservative estimates for Singapore from 2010-2050. BMC Public Health. 2016; 16:153. doi:I0.I |86/s I2889-016-2827-I.

2. Ndoro, Samuel. Effective multidisciplinary working: the key to high-quality care. Br J Nurs. 2014;23: 724-7.

doi:I0.12968/bjon.2014.23.13.724.

3. Schulz R, Sherwood PR. Physical and Mental Health Effects of Family Caregiving. Am J Nurs. 2008;108(9 Suppl):23-27. doi:I0.1097/0I.NAJ.0000336406.45248.4c. 


\section{LEARNING POINTS}

- Patients who are admitted after an acute event such as a stroke, tend to present with associated conditions or complications, e.g. post-stroke depression, that hinder their recovery or discharge back to the community. Family physicians need to proactively look out for such conditions and aggressively manage them so as to ensure better outcomes.

- SBAR4 Complex care model - Situation, Background, Assessment, Recommendation, Resource, Responsibility, and Relationship. SBAR4 provides a framework for managing a complex case with bio-psycho-social components. It is based on the SBAR model of clinical communication and Pendleton's 7 Tasks of consultation. The advantage of using SBAR4 is that it will assist the family physician to have an organised approach to complex care and to coordinate the care across the multidisciplinary team involved in the care of the patient.

- Frailty is a marker of poor functional outcomes. Sarcopenia is an important modifiable risk factor for frailty. Sarcopenia may be reversed with good nutrition and rehabilitation programmes to improve muscle mass and strength. Various community programs are available in the community to promote successful ageing, e.g. SPICE, NTUC Silver Circle for Day/Senior Care Centres. 